\title{
Soziale Dienste zwischen öffentlicher Kontrolle und privater Erbringung
} Die kommunale Daseinsvorsorge im Lichte der Europäischen Union

Peter Herrmann

Prof. Dr. Peter Herrmann ist

Direktor des privaten Instituts für Sozialforschung und Sozialberatung »Institute for Independent Social Research and Consultancy « in Irland.

Internet http://www.esosc.eu
Der Begriff der Daseinsvorsorge weist zwei Dimensionen auf: Zum einen geht es um ein spezifisches deutsches Verständnis für Dienste, deren Erbringung mit einer öffentlichen Verantwortung im $\mathrm{Zu}$ sammenhang steht; zum anderen spielen die »Dienste im allgemeinen Interesse " in der Europäischen Union eine bedeutsame Rolle.

In der europäischen Diskussion um die Dienste des Allgemeininteresses war es zunächst schwierig, einen gemeinsamen Sprachnenner zu finden. Denn was dem einen »Daseinsvorsorge « war, meinte der andere als "general interest « zu verstehen und für andere kam es in vieler Hinsicht dem »intérêt publique " gleich. Die Einigung auf den Begriff des Allgemeininteresses bedeutet nicht unbedingt, dass man damit auch bei dem gleichen Verständnis angelangt ist. Dabei ist es nicht die offensichtliche Frage, ob eine Überbrückung zwischen den verschiedenen Interpretationen möglich ist und wie diese aussehen kann. Vielmehr ist es die Frage, was denn überhaupt das Soziale an den sozialen Diensten ist.

\section{Soziale Qualität - worum es gehen könnte}

Im Zusammenhang mit der Arbeit an sozialer Qualität wird hier vorgeschlagen, als Kernfrage des Sozialen die produktiven und reproduktiven Beziehungen der Menschen zu verstehen. Damit ist als allgemeiner Bezugspunkt einerseits die krude materielle Lebensbewältigung gegeben, zugleich sind aber drei Beziehungsdimensionen in das Blickfeld gerückt: die des Einzelnen zu der »natürlichen Umwelt «, dies in einem weiten Verständnis; jene zu den Mitmenschen; und schließlich diejenigen zwischen verschiedenen Gruppen und Klassen zur bestehenden sozialen und natürlichen Umwelt in den jeweiligen Institutionen und Strukturen. Dabei geht es um Bedingungsgefüge (soziomaterielle Ressourcen etc.) und ebenso »Konstitutionen ", also bestehende Handlungsfähigkeiten und gegenseitige Anerkennungsmuster - und damit auch um eine aktive Rolle jedes Menschen in diesem komplexen Prozess.

Die direkte und ausdrückliche Einbeziehung der natürlichen Umwelt ist eine Änderung gegenüber früheren Definitionen; auch wenn es dabei nicht um einen prinzipiellen Wechsel geht, so ist doch hier die Orientierung auf die Umwelt klarer herausgearbeitet. Und dies macht es möglich, soziale Dienste im Rahmen der Überlegungen zu sozialer Qualität klarer zu verorten. Nun lässt sich herauszustellen, dass jeglicher Versuch, diese Dienste in der Tradition von Wohltätigkeit zu interpretieren, nicht vorwärtsweisend ist. Es geht darum, klare Kriterien zu entwickeln, in deren Rahmen soziale Dienste als personenorientierte soziale Dienste des Allgemeininteresses zu verstehen sind. Diese Definition stammt aus dem Projekt »Quality and Accessibility of Social Services for Inclusion ", das unter Leitung von Eurodiaconia, einem europäischen ökumenischen Verband von Kirchen, nichtstaatlichen Wohlfahrtsorganisationen und Nichtregierungsorganisationen, und mit Finanzierung durch die EU-Kommission durchgeführt wurde.

Personenorientierte soziale Dienste des Allgemeininteresses sind grundlegend durch ihre hybride Eigenschaften charakterisiert. Die folgenden Aspekte müssen in ihrer Erbringung - und der Definition - berücksichtigt werden:

- Soziale Dienste sind auf Personen ausgerichtet und zur gleichen Zeit auf das Soziale orientiert.

- Außerdem sind sie orientiert am »Dienen « und an der Teilhabe im sozialen und persönlichen Leben.

- Und sie sind befasst mit der Orientierung am Allgemeininteresse und dem persönlichen Lebensweg. 
(Eurodiaconia: Quality and Accessibility of Social Services for Inclusion. General report; Brussels, o.D. [Juni 2005]: S. 20.)

\section{Spannungen - und wie man sie abzubauen versucht}

Nun öffnet sich freilich im Zusammenhang mit der kommunalen Perspektive eine weitere Schere, denn wir haben es mit zwei Seiten von Nähe zu tun: einerseits das Erkennen persönlicher Bedarfe, lokaler Ressourcen zur Hilfe und emotionaler Nähe; andererseits eben auch der Kontrolle und normativen Bindung, damit die Forderung nach »Wohlverhalten $«$.

Dies mag zunächst als allgemeiner Hintergrund ausreichen und einen Rahmen bieten, die aktuellen Diskussionen in der Europäischen Union (EU) einzuordnen. Wenn auf »aktuelle Diskussionen hingewiesen ist, so ist festzustellen, dass diese eigentlich längst abgeebbt sind: EUoffiziell wurde eine solche Diskussion seit Mitte der 1990er Jahr geführt und es lässt sich sagen, dass sie vor zwei Jahren erfolgreich beendet wurde.

Erfolgreich bedeutet: Die Marktkräfte haben letztlich obsiegt. Wenn auch die Dienstleistungsrichtlinie, das Monti-Paket u. Ä. nicht vollständig in ihren ursprünglichen Fassungen angenommen wurden, so kam doch, der Geist, aus dem das kroch, weit genug aus seinem Loch: Bestimmte Gedanken sind eindeutig verankert und es ist weitgehend anerkannt, dass die tatsächlich bestehenden Probleme einer ineffizienten und mit Blick auf Qualitätssicherung nur unklar definierte und kontrollierte Dienstleistungserbringung nun in bestimmter Richtung angegangen werden. Es handelt sich also um Marktleistungen. Die formal vorhandene Anerkennung der Wichtigkeit der Sicherung von "Diensten des Allgemeininteresses « bedeutet nur, dass vage Sicherungen bestehen. Zum einen bleibt es den Mitgliedsaaten überlassen, was sie solchen Diensten zuordnen. Zum anderen wird betont, dass solche Dienste wichtig sind, ohne aber auf die Eigentumsformen und Organisationsweisen einzugehen.

Erhebliche Probleme ergeben sich daher in mancherlei Hinsicht: lokale und regionale Unterschiede; eine Orientierung auf Mindeststandards sowie eine Orien- tierung auf »kleine Einheiten«, denn messbar ist nicht wirklich »der Dienst « als komplexe Handlungs- und Interaktionseinheit, sondern nur die Vielzahl von einzelnen Handlungen und Aktionen. Prozesse gesellschaftlicher Praxis werden somit durch »Handlungsketten « ersetzt: Einzelne Aktionen und Aktivitäten scheinen wichtiger zu sein. Wichtig ist der damit entstehende indirekte Druck, denn die Rechtssituation ist in vielerlei Hinsicht nach wie vor schwer zu durchschauen, wenn es um die konkrete Bedeutung der Vorschriften und Anforderungen geht.

Die Nichtregierungsorganisationen und verbundene politische Kräfte sind eher verhalten und suchen die Antworten,
Formulierungen richtet sich auf immer neue Facetten, weil politisch offensichtlich nicht anerkannt werden darf, dass ein großer Schritt erforderlich ist.

\section{Gegenwart der Vergangenheit}

Der Ausdruck »Gutes zu tun « findet sich zwar nicht im offiziellen Jargon, drückt aber aus, dass es doch letztlich mit Blick auf das Soziale eben vage ist, was da von sozialen Diensten erwartet wird. Es ist eher immer wieder eine unausgesprochene Wiederbelebung von »Wohltätigkeits-Ideen « zu finden. Denn wann immer es um einschlägige Definitionen geht,

\section{"Die Europäische Union probt bei der Daseinsvorsorge den Spagat zwischen öffentlicher Kontrolle und privater Erbringung «}

die sie » wissen «, aber nicht so recht formulieren können (in diesem Zusammenhang eine Ausnahme: Informal Network of Social Services Providers Seminar "Impact of EU legislation on social services «. Brussels 29.9.2009; in: Herrmann, Peter: Social Services - Social Injustice through Empowering Customers and Users?; Bremen: Europäischer Hochschulverlag; i. V.). Oder ist es die Angst, die sie von Formulierungen zurückhält? Die Angst zum einen, zugestehen zu müssen, dass sie eigentlich so besonders nicht (mehr) sind, da sie längst vollständig in das Wettbewerbssystem eingebunden sind - eingebunden wurden und sich selbst in dieses Geflecht eingelassen haben?

Ein Teil der Europäischen Kommission - der "Sozialbereich « - sitzt ein wenig zwischen den Stühlen und besteht darauf, einerseits den Vermarktungsdruck zu akzeptieren, andererseits darauf, dass es in dem bestehenden vagen gesetzlichen Rahmen schon möglich ist, » Gutes zu tun«. Und bei Projekten ist eher eine Verzögerung und Überbrückung der Unsicherheiten zu sehen, als eine wirklich sich anbahnende Lösung; etwas, was Insider aus der EU-Armutsbekämpfung kennen dürften: Die Suche nach Lösungen und neuen finden sich diese auf einer anderen Ebene: So weist der Begriff der Daseinsvorsorge zwei Dimensionen auf. Es geht um das spezifisch deutsche Verständnis von Diensten, deren Erbringung mit einer öffentlichen Verantwortung im $\mathrm{Zu}$ sammenhang steht; zum anderen geht es weitergehend um »Dienste im allgemeinen Interesse « im Sinne der Europäischen Union.

Der Begriff Daseinsvorsorge wurde Ende der 1930er Jahre von Ernst Forsthoff eingeführt. Es handelt sich um einen verwaltungsrechtlichen Begriff, nach dem es um »die Darbringung von Leistungen, auf welche der in die modernen massentümlichen Lebensformen verwiesen Mensch lebensnotwendig angewiesen ist « (Forsthoff, Ernst: Die Verwaltung als Leistungsträger. Kohlhammer, Stuttgart 1938: S. 7).

Damit weist der Begriff eine starke wohltätigkeitsstaatliche und ebenso eine paternalistische Dimension auf. Wohlfahrtstätigkeit bezieht sich auf ein breites Verständnis und bezieht nicht zuletzt auch sogenannte Netzwerkindustrien und Energieversorgungsleistungen ein. Dabei ist zu berücksichtigen, dass Forsthoff selbst mit dem Begriff eine starke 
wirtschaftslenkende Rolle verbindet. Durch die verwaltungsrechtliche Fassung des Begriffs und seine Einbindung in den Zusammenhang deutscher Rechts- und Verwaltungsstrukturen kommt der Kommunalebene sowie dem Subsidiaritätsprinzip eine besondere Rolle zu. Zwar wird die staatliche Verantwortung ausdrücklich hervorgehoben. Da die Erbringung der Dienste der Daseinsvorsorge aber unmittelbar vor Ort erfolgt und im Zusammenhang mit der Wirtschaftslenkungsfunktion regionale Unterschiede eine starke Rolle spielen, ist die lokale Ebene besonders gefordert.

Schaut man sich die tatsächliche Lage an, so zeigen gerade die Debatten der letzten Jahre, dass das Konzept immer noch offen und nach nationalen Traditionen umstritten ist. In diesem Sinne ist die vage Definition in den Kommissionsdokumenten letztlich hilfreich: Eine geistig-moralische Wende ist wichtiger und faktisch entscheidender als ein formaler Rahmen letzterer würde gar Versuche ermutigen, nach definitiven Rechts(aus)wegen zu suchen.

So gilt mittlerweile im EU-Jargon schlicht eine Einigung auf den Begriff der Dienste des Allgemeininteresses. Im Vertrag von Nizza lautet es in Art. 16: »Un- beschadet der Art. 73, 86 und 87 und in Anbetracht des Stellenwerts, den Dienste von allgemeinem wirtschaftlichem Interesse innerhalb der gemeinsamen Werte der Union einnehmen, sowie ihrer Bedeutung bei der Förderung des sozialen und territorialen Zusammenhalts tragen die Gemeinschaft und die Mitgliedstaaten im Rahmen ihrer jeweiligen Befugnisse im Anwendungsbereich dieses Vertrags dafür Sorge, dass die Grundsätze und Bedingungen für das Funktionieren dieser Dienste so gestaltet sind, dass sie ihren Aufgaben nachkommen können."

Erprobt wird damit ein Spagat zwischen öffentlicher Kontrolle und privater Erbringung. Es handelt sich darum, dass bestimmte Kriterien, die gemeinhin mit öffentlicher Verantwortung gesehen werden - Solidarität, soziale Kohäsion, soziale Integration - mit den Kriterien privater Profiterbringung konzeptionell verbunden werden sollen.

\section{Mögliche Zukunft}

Sicher hat der vage Charakter vieler Definitionen und Vorschriften auch Vorteile: Unbestimmtheit ist auch eine offene Tür für diejenigen, die nach Spielräumen suchen, das Soziale in einem handlungsoffenen und emanzipierenden Verständnis zu realisieren. Dazu gehört, die Dienstenutzer nicht allein als Kunden, sondern vor allem als Bürger zu sehen. Es geht nicht als Erstes um Konsumentenrechte, sondern um Bürgerrechte. Dies bedeutet auch, dass entsprechende Dienste und ihre Qualitätsstandards nicht Lieferstandards sind, sondern: Kommunalstandards.

Die Kommunen müssen sich wieder mehr in ihre Rolle als öffentliche Einheiten begreifen und sich selbst nicht allein oder schwerpunktmäßig als Verwaltungseinheiten verstehen. »Kommunal « sollte im Selbstverständnis in den Vordergrund stellen, dass es eben um Gemeinsames geht und darum, etwas für das Gemeinsame zu tun. Dem steht der derzeitige Kurs entgegen. Denn selbst dort, wo allgemein an einem hohen Standard festgehalten wird, wie in den nordischen Ländern, ist nicht von der Hand zu weisen, dass etwa durch die Auslagerung oder durch Public-private-Partnerships eine Orientierung auf eine Privatmentalität hervorgehoben wird - anstatt soziale Dienste als Bürgerrechte in einem speziellen Produktions- und Reproduktionsbereich zu begreifen.

\section{Daseinsvorsorge in der Europäischen Union}

Was man heute unter »Daseinsvorsorge « versteht. Den Begriff der Daseinsvorsorge prägte Forsthoff 1938. Er verstand darunter die Gesamtheit der Leistungen der Verwaltung zur Befriedigung der Bedürfnisse der Bürgerinnen und Bürger für eine normale, den jeweiligen Lebensstandard entsprechende Lebensführung. Üblicherweise erbrachten die Kommunen diese Leistungen als klassische Angelegenheiten der örtlichen und überörtlichen Gemeinschaft. So wurden traditionell beispielsweise Versorgungs- und Verkehrsleistungen, Gesundheitspflege und Bildungseinrichtungen geschaffen. Kommunen konnten mangels Konkurrenz in diesen Bereichen oft marktfern agieren. Der Begriff Daseinsvorsorge markiert gleichzeitig den Übergang von der Eingriffs- (d. h. Gefahrenabwehrverwaltung) hin zur Leistungsverwaltung. Er ist jedoch kein Rechtsbegriff. Grundsätzlich wird die Versorgung der Bürgerinnen und Bürger mit notwendigen Dienstleistungen und Gütern im Rahmen der sozialen Marktwirtschaft nicht als Aufgabe des Staates betrachtet. Der Staat trägt jedoch die Verantwortung dafür, dass die Rahmenbedingungen entstehen, die die Erfüllung dieser Bedürfnisse durch Private ermöglichen. Daseinsvorsorgegüter und -dienstleistungen werden in Deutschland durch öffentliche und private Akteure erbracht.
Dabei wird die staatliche Pflicht zur Daseinsvorsorge zunehmend durch Gestaltung der Rahmenbedingungen von Leistungen zur Daseinsvorsorge durch eigene Verwaltungseinrichtungen, Eigenbetriebe oder staatliche oder kommunaleigene Unternehmen sowie in Kooperation mit Privaten in gemischt wirtschaftlichen Unternehmen, wenn die Erbringung nicht vollständig durch Private erfolgt. Wohl unter dem Druck der Europäischen Union (EU) wird auch hier zunehmend marktorientiert verfahren. Auf europarechtlicher Ebene besteht ein Abstimmungsbedarf mit dem Begriff der Dienstleistungen von allgemeinem wirtschaftlichen Interesse (Art. 86 Abs. 2, 16 Vertrag zur Gründung der Europäischen Gemeinschaft). Dieser Begriff entscheidet über die Anwendung von europäischen Wettbewerbsregeln auf das betreffende Unternehmen. Eine Tätigkeit ist als wirtschaftlich einzuordnen, wenn Waren oder Dienstleistungen auf einem bestimmten Markt angeboten werden. Ein allgemeines Interesse liegt grundsätzlich vor, wenn die Tätigkeit mit besonderen Gemeinwohlverpflichtungen verbunden ist bzw. wenn sie im Interesse der Allgemeinheit liegt. Das Unternehmen muss mit der fraglichen Tätigkeit durch Hoheitsakt betraut werden. Als Beispiele für solche Dienstleistungen von allgemeinem wirt- 
schaftlichen Interesse sind von EU-Kommission und Gerichtshof der EU u. a. anerkannt worden: Postdienste, Stromversorgung und Arbeitsvermittlung.

Angela Busse Prof. Dr. Angela Busse lebrt Sozialrecht an der Hochschule Fulda.

Welche Bedeutung »Sozialdienstleistungen von allgemeinem Interesse « in Europa haben. Der Rechtsbegriff entstammt teilweise dem Vertrag über die Arbeitsweise der Europäischen Union (AEUV). Er setzt sich zusammen aus »sozialen Diensten « und "allgemeinem wirtschaftlichen Interesse « nach den Art. 14, 106 Abs. 2 AEUV, ex-Art. 16, 86 Abs. 2 EGVertrag. In Deutschland wird das allgemeine Interesse mit Daseinsvorsorge, Gemeinwohl- bzw. Gemeinweseninteresse oder Gemeinnützigkeit gleichgesetzt. Europarechtlich stellt es seit der Aufnahme des Begriffs in den Vertrag von Amsterdam (1997) ein Abgrenzungsmerkmal zu rein gewerblichen, gewinnorientierten Tätigkeiten eines Anbieters von Dienstleistungen dar. Die Differenz zwischen der deutschen und europäischen Definition führt insbesondere mit Blick auf die bereits liberalisierten Bereiche der Sozialmärkte in Deutschland, z. B. in der Pflege, Kinderbetreuung, zu Fragen bei der Anwendung des europäischen Wettbewerbsrechts, z. B. Privilegierung im Rahmen des Beihilferechts. Die Europäische Kommission hob in einer Mitteilung zu den »Sozialdienstleistungen von allgemeinem Interesse « 2006 deren Besonderheiten hervor, verwendet den Begriff aber seit 2007 nur noch zurückhaltend.

Cornelia Markowski

Cornelia Markowski ist Leiterin der Stabsstelle Internationales im Deutschen Verein für öffentliche und private Fürsorge e. V.

Wie die Sozialwirtschaft in Europa ihre Interessen organisiert. CEDAG - das Europäische Komitee der Verbände von allgemeinem Interesse (Comité Européen des Associations d'intérêt Général) wurde 1989 gegründet, um Anliegen der Sozialwirtschaft auf europäischer Ebene zu vertreten und den gemeinnützigen, nicht gewinnorientierten Vereinen und Verbänden eine Stimme zu geben. CEDAG hat u. a. zum Ziel, den Beitrag freier Vereine zur sozialen und kulturellen Entwicklung aufzuzeigen, die Anerkennung des Vereinssektors im Hinblick auf ihren wirtschaftlichen Beitrag und ihr gemeinwohlorientiertes Handeln zu fördern, Gemeinschaftsmaßnahmen anzuregen, die zur Entwicklung und Förderung des gemeinnützigen Sektors beitragen, den gegenseitigen Erfahrungs- und Informationsaustausch zu fördern und zu unterstützen, mit anderen europäischen Organisationen zusammenzuarbeiten und nationale Plattformen und Dachorganisationen zusammenbringen, die Einbeziehung der Vereine in den zivilen und sozialen Dialog auf europäischer Ebene zu fördern. Schwerpunkte der Tätigkeit sind die Sicherung der Daseinsvorsorge, die Schaffung eines europäischen Rahmens für soziale Dienste, die Politik der sozialen Eingliederung sowie Fragen der Sozialwirtschaft. CEDAG möchte zudem den "zivilen « Dialog zwischen Nichtregierungsorganisationen und den nationalen Regierungen bzw. den Institutionen der Europäischen Union (EU) fördern und setzt sich etwa für eine Europäische Charta des zivilen Dialogs ein. Derzeit hat CEDAG 32 Mitglieder aus 17 EU-Mitgliedstaaten und vier aus Nicht-EU-Ländern. CEDAG ist Mitglied der Platform of European Social NGOs, einem Zusammen- schluss von rund 40 europäischen Netzwerken. Informationen: Internet http://www.cedag-eu.org.

Ulrich Tiburcy

Ulrich Tiburcy ist Leiter der EU-Vertretung der Bundesarbeitsgemeinschaft der Freien Wohlfahrtspflege in Brüssel.

Wer Informationen zur Sozialwirtschaft in Europa sammelt und aufbereitet. Politik und Rechtsetzung der Europäischen Union werden zunehmend für die sozialwirtschaftlichen Akteure strategisch und operativ relevant. Zugang zu den entsprechenden Informationen bietet EUFIS, das internetbasierte EU-Fach- und Förderinformationssystem der Bank für Sozialwirtschaft. EUFIS bietet zeitnah Informationen zur EU-Politik, zur EU-Rechtsetzung, zur EU-Förderung, zu Entscheidungen der EU-Institutionen sowie zu den Europaaktivitäten nationaler Akteure der Sozial- und Gesundheitswirtschaft. Das speziell auf die Sozial- und Gesundheitswirtschaft fokussierte Informationsspektrum umfasst insbesondere die Themenbereiche Soziales, Gesundheit, Beschäftigung, Forschung, Bildung, Jugend, Kultur, Migration, Menschenrechte, Entwicklungszusammenarbeit sowie Regional- und Stadtentwicklung; hinzukommen für die Sozialwirtschaft relevante Querschnittsthemen wie z. B. Daseinsvorsorge, Binnenmarkt und Steuerrecht. Diese Informationen werden in EUFIS als tagesaktuelle Nachrichten geliefert, ein Wochenplan gibt einen Überblick über alle für die Sozialwirtschaft relevanten Themen, die in den EU-Institutionen in der laufenden Woche auf der Tagesordnung stehen. EU-Dokumente (Kommissionsmitteilungen, Berichte und Stellungnahmen der EU-Organe, Studien und die EU-Rechtsetzung etc.) werden unmittelbar zugänglich gemacht. Informationen zu EU-Fördermaßnahmen sowie die Hintergrunddokumente sind abrufbar; ergänzend gibt es einen Antragskalender, der auf aktuell laufende Fristen zur Einreichung von Förderanträgen hinweist. Erfasst werden auch Tagungen und Kongresse über europapolitische Themen. Ein Adressenpool erleichtert das Auffinden nationaler und europäischer Ansprechpartner. Ausgewählte aktuelle europäische Themenstränge sind in Dossiers konzentriert zusammenhängend aufbereitet. Informationen: Internet http://www.eufis.de.

Berthold Becher Dr. Berthold Becher ist Leiter der Abteilung Research, sozialwirtschaftliche Marktunterstützung, Europa-Service der Bank für Sozialwirtschaft AG.

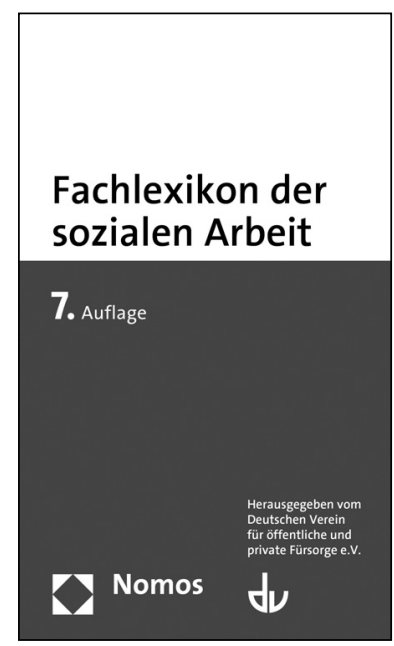

Quelle: Deutscher Verein für öfentliche und private Fürsorge e. V. (Hg.): Fachlexikon der sozialen Arbeit. 7. Auflage. Nomos Verlagsgesellschaft, Baden-Baden 2011. Ca. 1.200 Seiten. 44,- Euro. ISBN 978-38329-5153-5. 
"Da es nicht für alle reicht, springen die Armen ein."

Ernst Bloch, deutscher Philosoph (1885-1977)

"Geld ist nicht alles - mit zwanzig Millionen Dollar kann man genauso glücklich sein wie mit einundzwanzig."

Donald Trump, amerikanischer Milliardär (geb. 1946)

„Es gibt viele Wege, wie Gesellschaften ihre Chancen verpfuschen können, hingegen gibt es nur wenige, die zu Wohlstand führen."

Partha Dasgupta, indisch-britischer Wirtschaftswissenschaftler (geb. 1942)

"Die Phönizier haben das Geld erfunden - aber warum so wenig?" Johann Nepomuk Nestroy, österreichischer Schriftsteller (1801-1862)

"Von jetzt an werde ich nur noch so viel ausgeben, wie ich einnehme -

selbst wenn ich mir dafür Geld borgen muss."

Mark Twain, amerikanischer Schriftsteller (1835-1910)

"Wachstum ist nur dann gesund, wenn es sich innerhalb einer Struktur vollzieht. " Erich Fromm, deutsch-amerikanischer Psychoanalytiker (1900-1980)

„Noch einmal sprechen / von der Wärme des Lebens /

damit doch einige wissen: / Es ist nicht warm /

aber es könnte warm sein"

Erich Fried, österreichischer Schriftsteller (1921-1988) 